\title{
Encephalitis - when viral infections attack the brain
}

Professor Clayton Wiley from the University of Pittsburgh studies the emergence of new brain viral infections and their link to dementia. an inflammation of the brain tissue that disrupts brain function and causes symp of variable severity, such as headache, fever, confusion, a sense, memory problems, trouble focusing or speaking, or even seizures. Knowledge of these viruses which manage to mitigate infection and to prepare for the inevitable arrival of newly emergent and unknown infectious agents. altered consciousness, loss of

It took humans millions of years to evolve and become who we are today. we are already familiar with can evolve we are already familiar with can evolve the virus responsible for the ann, Humans have learned to cope with those. Sometimes, however, new viruses emerge and take us by surprise, and some of them can even disrupt our daily lives by causing pandemics - something we know all too well in 2021

\section{WHAT ARE VIRUSES?} A virus is made of genetic material (DNA or RNA) contained inside a protective envelope with proteins on its surface. To survive and thrive, it needs a host - for example, a human. Once the host is infected, the virus exploits the host's cell machinery to replicate. To do that, the cell. The vira genetic anderia that indicates how to synthesis a code component of the virus Inside the host cell, thousands of envelope proteins and copies of the genetic material are synthesised and assembled into new viral particles. These viral particles are

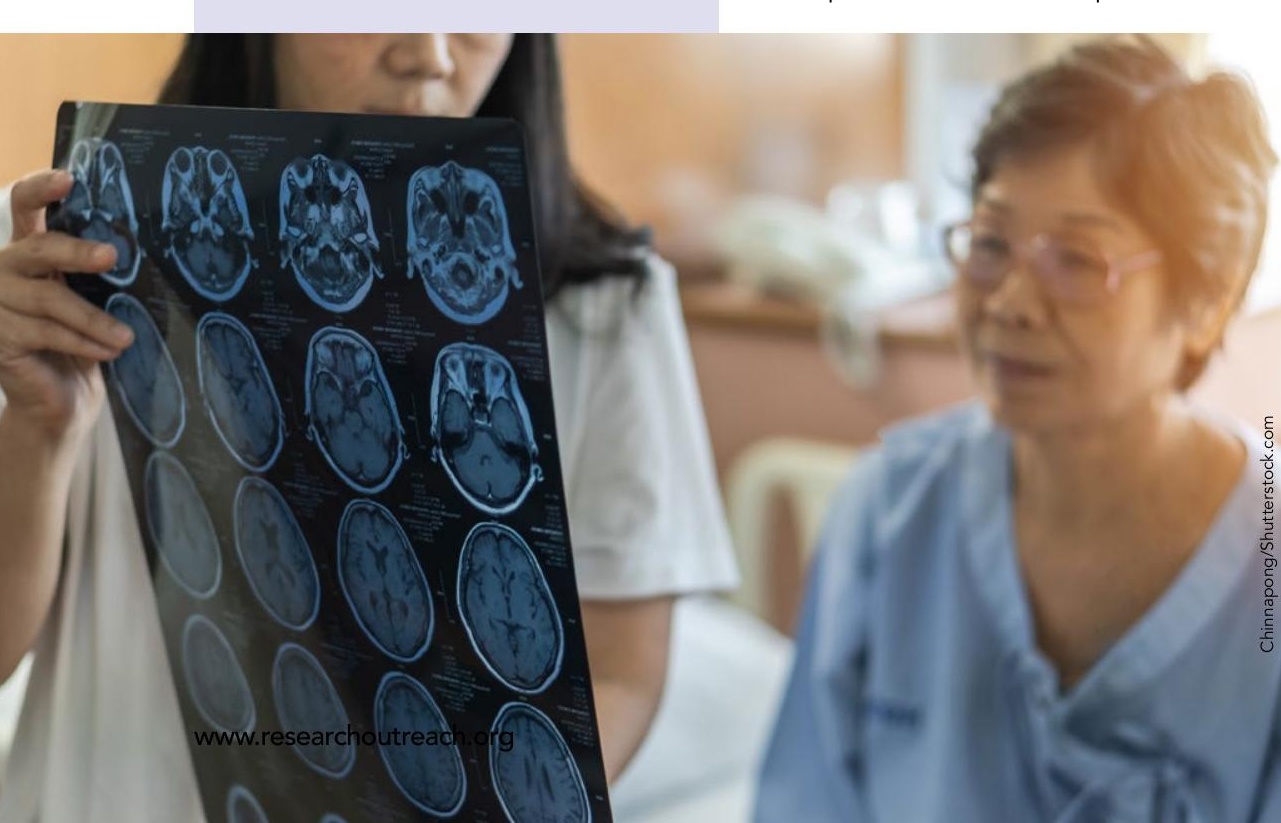

then released to spread within the host The firl sic cells to keep replicating. another host.

A broad range of strategies enable ransmission from one host to another example, replicate mostly in the host gut, and rely upon hand-to-mouth contact to infect a new host. Other viruses called arboviruses are found in blood. Their transmission is facilitated by vectors such

\section{OUR DEFENCE MECHANISMS} Dnate and adaptive immunity are host defence mechanisms that prevent viruses from growing inside of our cells.

Innate immunity includes physical and biochemical barriers. Skin, for examp first line of defence againstinvading pathogens (viruses, bacteria). Mucus in the nose is another example of a barrier: it can trap viruses in the air we breathe. Innate immunity also has a role to play If viruses manage to enter the body despite the physical barriers. Viruses are recognised as foreign elements by host cells. This recognition triggers the destruction of the pathogen.

Adaptive immunity involves molecules such as cytokines and antibodies which neutralise viruses, and cells which kill infected host cells. The adaptive immune system has a memory. it somembers initial pathogen infections protective response is more robust and effective.

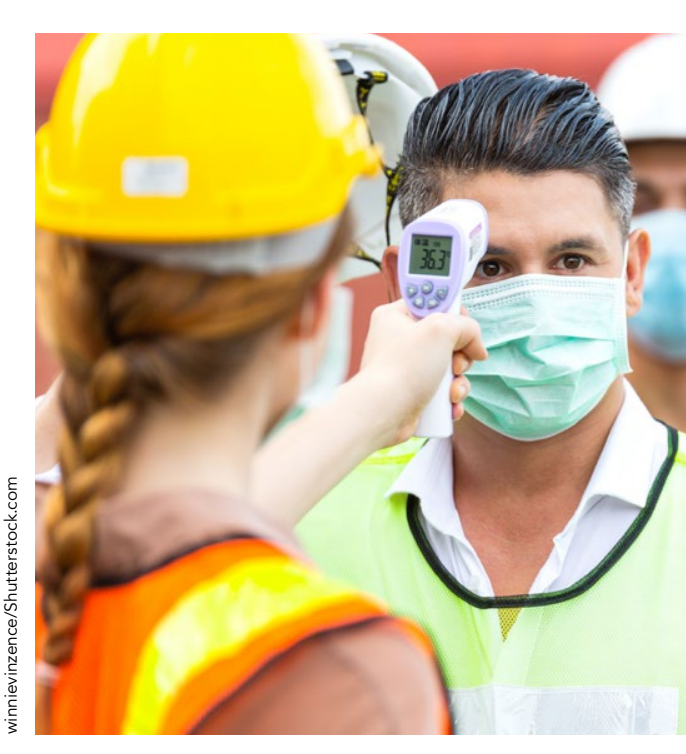

THERAPEUTICS

Though we have developed powerful defence mechanisms, some viruses When that is the case, therapeutics a needed to boost our immune defences. An ideal antiviral drug is a drug that specifically disrupts an element or physiological mechanism which is essential to the virus but not involved in host metabolism, so that it targets the virus without
causing harm to the host. Such drugs are difficult to discover and, sometimes, the best therapy to treat viral infection is to prevent it from
occurring in the first place. That is when occurring in the first place. That is when

PIDEMICS AND PANDEMICS A common way for viruses to evade the inmune system is to change the proteins this through mutations. When enough mutations accumulate, the viral protei changes so much that host antibodies are unable to recognise it. The virus then escapes neutralisation by the immune system. This leads to a new epidemic.

From time to time, viruses create even greater havoc. when two different viruses infect the same cell, fragments of the genetic material can be exchanged. As they replicate and viral particles are assembled, entirely new reassortments are generated: the newly assembled
viral particles contain proteins from both viral particles contain proteins from bo
viruses. If these new viruses are capable

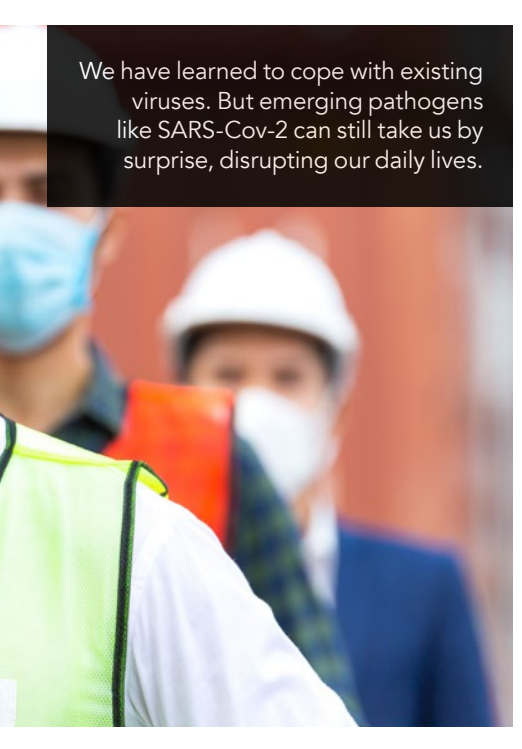

of efficient transmission, they can be the origin of a new pandemic.

\section{BRAIN BARRIERS}

Protecting the brain from viruses and other pathogens is of the utmost importance. created an amazing set of barriers that prevent viruses from reach This begins with hair on the scalp, orming an insulating layer of air above

Natural selection has created an mazing set of barriers that prevent iruses from reaching the brain.

impermeable skin surface. Beneath the skin, the skull and additional tissue layers named meninges protect the brain. With introduced directly into the brain.

Brain infection occurs only when

pathogens are present elsewhere in the body. Then, they can spread to the brain some barriers. Blood vessels in the brain

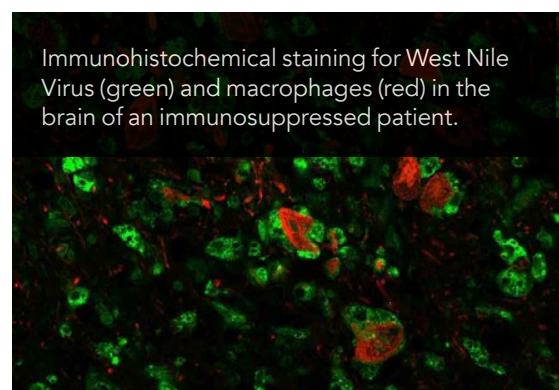

pandemic threats: to who have been in close, only humant with infected birds develop lethal neurologica disease caused by Avian $\mathrm{fl}$, because the be transmitted from human to hum However, this may not last indefinitely.

WEST NILE VIRUS West Nile virus is transmitted to humans symptoms in up to $80 \%$ of infected
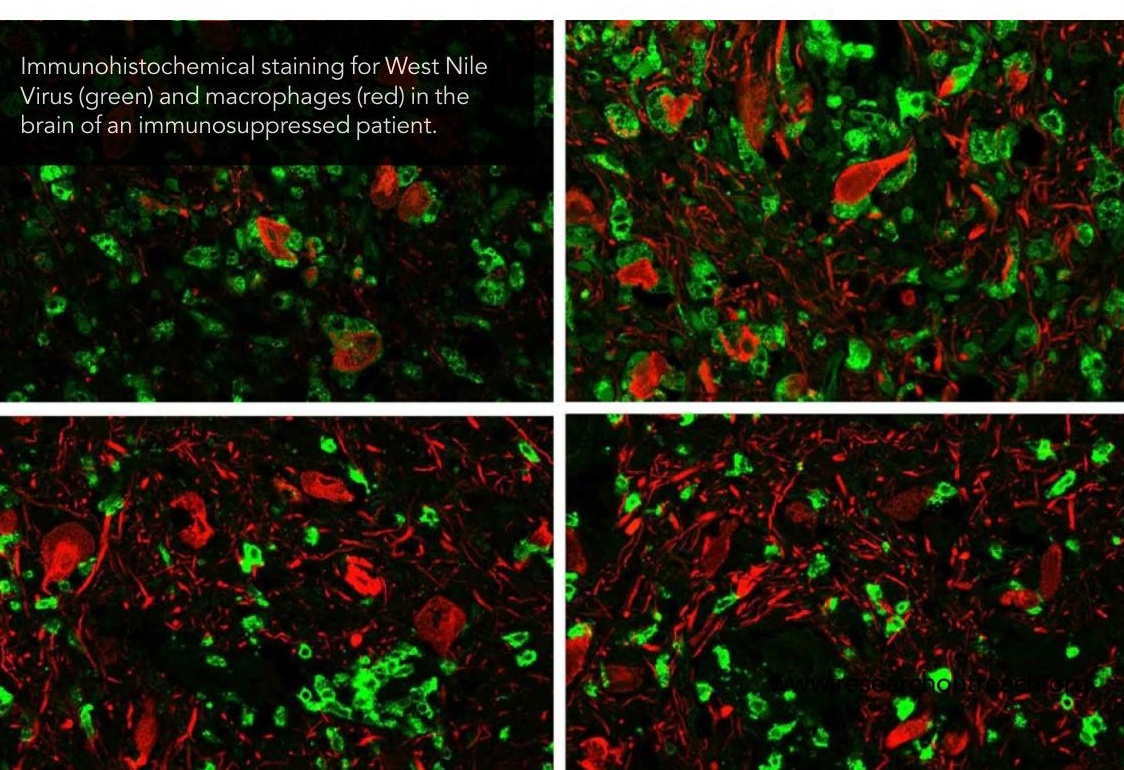

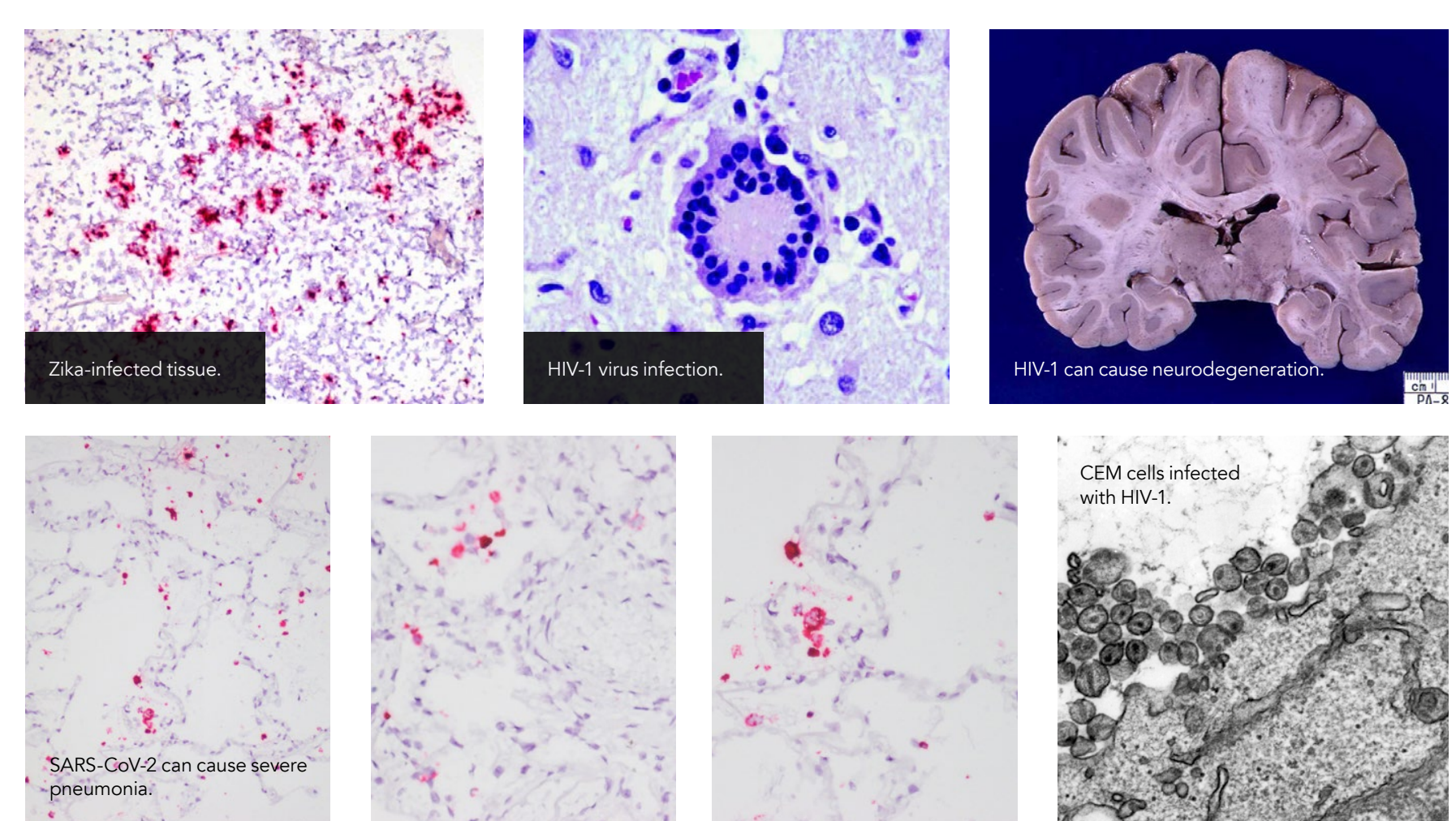

humans, the remaining 20\% experience classical symptoms of encephalitis, such as fever, headache, fatigue, malaise, muscle pain, and weakness. When the virus spreads to the brain, it can cause disorientation, movement disorders paralysis, or even coma and death.

\section{ZIKA VIRUS}

Zika virus is also transmitted by mosquitoes. Since the 1950s, when Zka virus was first detected in a human, the virus has been ciculating widely in equat 2015, Zika virus spread to the Americas and populations with naive Anmune

systems unacquainted with the virus. Up

to one million infections were reported Brazil. While most infections are clinically mild, Zika virus can cause Guillain-

Barré syndrome, a serious condition of the nervous system characterised by numbness, weakness and pain in the feet, hands and limbs. Zika virus can also be transmitted from mother to foetus during the first trimester of pregnancy, resulting in microcephaly (abnormally small head size) and oht congental malformation in the infant.

HUMAN IMMUNODEFICIENCY VIRUS (HIV)

HIV-1, responsible for the AIDS epidemic, is derived from a chimpanzee agent which, about 70 years ago, evade the immune system, the virus is present in high quantity within the host over a long period of time, during which the host can spread it to new hosts through close contact exchang of blood or bodily secretions. This is how HIV-1 eventually infected over 70 million humans.

When HIV-1 enters the brain, it targets microglia, the sentinel

been transmitted to humans through intermediate hosts: palm civets for severe acute respiratory syndrome CoV respiratory syndrome CoV (MERS-COV), and possibly pangolins for SARS-CoV-2 (also known as COVID-19). All three of these CoVs mediate severe pneumonia and their appearance in humans was unanticipated. While SARS-CoV and MERS-CoV had limited spread (each causing about 800 deaths in the world), for more has been disrupting our lives for more than a year. Symptoms such as

Prof Wiley reviewed emergent viral infections and the neuropathology they caused in the past $\mathbf{5 0}$ years.

Infected microglia disrupt the brain and cause neurodegeneration (the progressive loss of neurons) and dementia.

CORONAVIRUSES

There are seven known coronaviruses (CoVs). Four of them are endemic, among humans. For most people, known human Covs are suspected not directly infect) the human brain. (SARS-CoV), camels for Middle East which means that they are widespread they just cause a cold. The three other and smell, or even loss of consciousness, seizures and strokes, show that COVID-19 affects (though probably does

Because SARS-CoV-2 has emerged recently, its long-term effects on cognition are still unknown, but researchers around the world are actively studying the disease. Could COVID-19 and therefore be linked to denelin? and therefore be will tell.

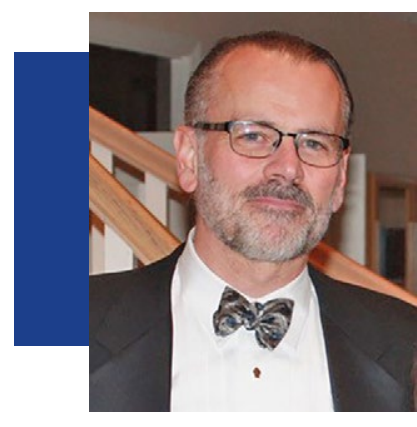

\section{Behind the Research} Prof Clayton A. Wiley

E: wileyca@upmc.edu T: +1 412-624-0764 F: +1 412-647-5610

W: www.neuro.pathology.pitt.edu/people/wiley W: https://vidementia.com/speaker/Clayton-Wiley

\section{Research Objectives}

Prof Wiley investigates the emergence of new brain viral infections and their link to dementia.

\section{Detail}

Address

200 Lothrop Street

Pittsburgh, PA 15213

USA

Bio

Clayton Wiley received his MD and PhD from University of California San Diego (UCSD) in 1981. After a pathology residency and neuropathology fellowship, he became Assistant Professor and later Professor at UCSD. Since 1993, Prof Wiley works at the University of Pittsburgh, where he is Director of the Division of Neuropathology. In addition, Prof Wiley directs the Neuropathology fellowship program and runs an $\mathrm{NiH}$-funded research program studying neuroinflammation and viral encephalitis in particular.

Funding
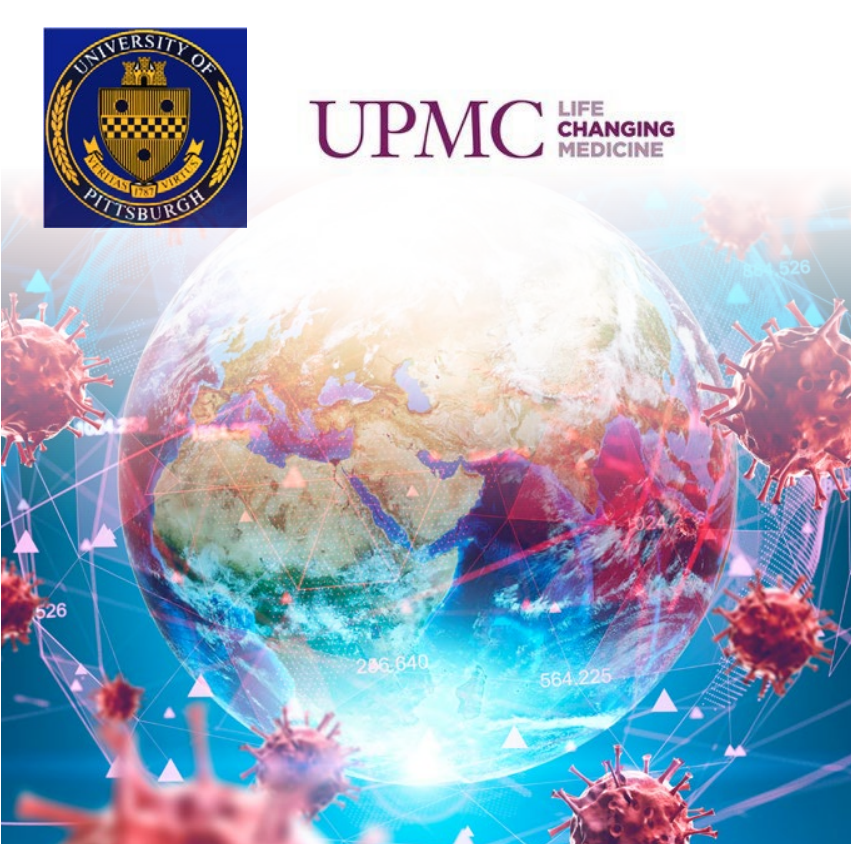

\section{References}

Wiley C. A. (2020). Emergent Viral Infections of the CNS. Journal of neuropathology and experimental neurology, 79
nlaa054

Bissel, S. J., Wang, G., Carter, D. M., Crevar, C. J., Ross, T. M. \& Wiley, C. A. (2014). H1N1, but not H3N2, influenza A virus infection protects ferrets from H5N1 encephalitis. Journal of virology, 88(6),
JVI.01840-13

Wiley, C. A., Bhardwaj, N., Ross, T. M., \& Bissel, S. J. (2015). Emerging Infections of CNS: Avian Influenza A Virus, Rift (Zurich, Switzerland), 25(5), 634-650. httros://doi.org/10.11111 bpa.12281

Bissel, S. J., Auer, R. N., Chiang, C. H., Kofler, J., Murdoch, G. H., N Nix, W. A., Painter, M., Richer, M., Sartelet, H., Wang, G., \&Wily, C. A. (2015). Human Parechovirus 3 Meningitis and Fatal Leukoencephalopathy. Jounnal of neuropathology org/10.1097/NEN.00000000000000215

Wiley, C. A., \& Chimelli, L. (2017). Human Zika and West Nile virus neurological infections: What is the difference? Neuropathology: official journal of the Japanese Society or New

Armah, H. B., Wang, G., Omalu, B. I., Tesh, R. B., Gyure, K. A., Chute, D. J., Smith, R. D., Dulai, P., Vinters, H. V.,
Kleinschmidt-DeMasters, B. K., \& Wiley, C. A. (2007). Systemic distribution of West Nile virus infection: postmortem immunohistochemical study of six cases. Brain pathology (Zurich, Switzerland), 17 (1),

\section{Personal Response}

\section{What inspired you to conduct this review?}

II With a global human population of almost 8 billion, we are an easy target for new viruses emerging from other species. As sadly proven by the COVID-19 pandemic, we infectious agents. But we also need to understand the virus life cycle and our immune response to it so we can devise
measures to prevent viruses from infecting our brains. 